\title{
Study of start-up decoupling controller for modular multilevel converter
}

\author{
Xinlin Zhang ${ }^{1}$, Yanchi Zhang ${ }^{1, *}$, Da Xie ${ }^{2}$ and Bowen Zhao \\ ${ }^{1}$ School of Electrical Engineering, Shanghai Dianji University, 201306 Shanghai, China \\ ${ }^{2}$ School of Electronic Information and Electrical Engineering, Shanghai Jiaotong University, 200240 Shanghai, China
}

\begin{abstract}
Modular multilevel converters are widely used in power systems because of their significant advantages. In this paper, a dynamic mathematical model is established by analyzing the topology, operating principle and switching function of the modular multilevel converter, so as to construct an inner and outer loop decoupling controller in dq coordinates. The carrier phase-shift pulse-width modulation is selected to control the sub-module operation, and the uncontrolled pre-charging method is adopted to charge the sub-module. Finally, simulation experiments are carried out in MATLAB/Simulink, and the results show that the control method achieves smooth start-up of the modular multilevel converter.
\end{abstract}

\section{Introduction}

Modular multilevel converter (MMC) as a new type of converter has many advantages such as high level number, low harmonic content, low switching losses, and simple redundancy expansion [1]. As the number of levels increases, the output voltage waveform can be improved, thus reducing the voltage waveform distortion and harmonic content. High frequency pulse width modulation (PWM) is widely used to trigger two-level and three-level converters, MMC can reduce the switching frequency and thus reduce the switching loss to a large extent. The MMC has multiple sub-modules for easy expansion and can be set up for redundancy to replace the faulty module in case of system failure without affecting the overall system operation. The expansion and redundancy of sub-modules also facilitate the setting of control methods such as protection control, capacitor voltage balancing, etc.

The research hotspots of MMC mainly include topology, modulation technology, capacitor voltage balancing, circulating current suppression, start-up control, etc. The practical applications are mainly DC transmission converter stations, reactive power compensation devices, new energy storage systems, etc. The literature [2] discusses the sub-module pre-charging method during MMC start-up, and proposes an AC-side start-up control method that can suppress inrush current. In the literature [3], a distributed control architecture scheme is proposed to improve the traditional centralized control strategy and experiments are conducted to obtain the experimental results for start-up, steady-state, and transient states. The literature [4] analyzed the MMC start-up process in detail in terms of models and control methods.
In this paper, the dynamic mathematical model is derived from the topology, operating principle and switching function of the modular multilevel converter. The decoupling controller based on $\mathrm{dq}$ coordinates is established by the obtained mathematical model. The trigger pulse of each sub-module is obtained by the decoupling controller. And then the control function of the converter is realized according to the carrier phase shift pulse width modulation (CPS-PWM). The simulation experiment selects the uncontrolled charging method, and charges the sub-module to a certain value. Start-up of the MMC is performed by decoupling controller. The MMC DC side voltage, sub-module voltage, bridge arm current, $\mathrm{AC}$ side reactive power and $\mathrm{AC}$ system three-phase current reach the target value.

\section{MMC basic theory}

\subsection{MMC topology}

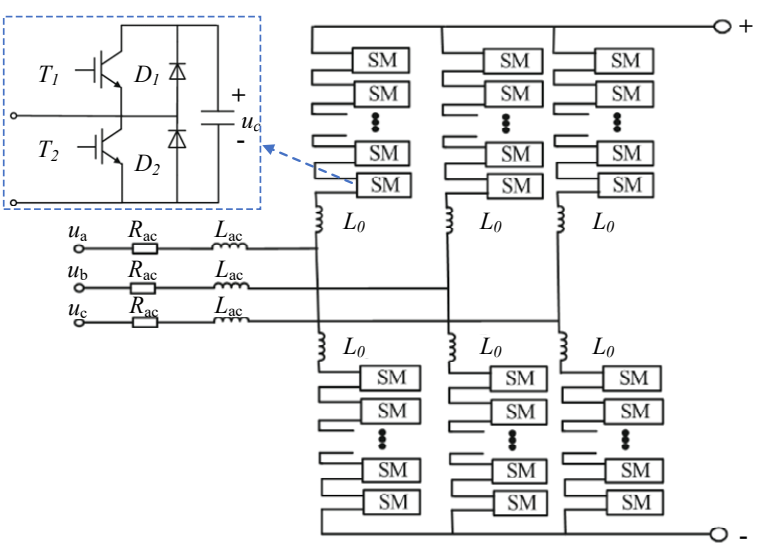

Fig. 1. MMC topology diagram

* Corresponding author: zhangyc@sdju.edu.cn 
The MMC topology is shown in Figure 1. The MMC consists of three phase cells, each phase cell includes two bridge arms, upper and lower, respectively. Each bridge arm includes $N$ sub-modules and a bridge arm inductor $L_{0}$. An inductor $L_{a c}$ and a resistor $R_{a c}$ are connected to the AC side of the MMC.

\subsection{MMC working principle}

The operating state of the MMC sub-module is switched by controlling the triggering and turning off of $T 1$ and $T 2$ in the sub-module. A five-level operating system, each phase unit includes 8 sub-modules, and the upper and lower bridge arms include 4 sub-modules respectively. The sum of the number of sub-modules put into the upper and lower bridge arms is constant at 4 , and the sum of the voltage of the upper and lower bridge arms is constant at $U_{d c}$, thus maintaining the DC side voltage constant. Then the three-phase AC voltage is regulated by the distribution of the number of sub-modules put into the upper and lower bridge arm.

Table 1. Sub-module input patterns for different time periods of the five-level MMC system

\begin{tabular}{|c|c|c|c|c|c|}
\hline Time & $U_{p}$ & $N_{-}$upper & $U_{\_ \text {upper }}$ & $N_{\_}$lower & $U_{\_}$lower \\
\hline$t_{0} \sim t_{1}$ & 0 & 2 & $U_{d c} / 2$ & 2 & $U_{d c} / 2$ \\
\hline$t_{1} \sim t_{2}$ & $-U_{d c} / 4$ & 3 & $3 U_{d c} / 4$ & 1 & $U_{d c} / 4$ \\
\hline$t_{2} \sim t_{3}$ & $-U_{d c} / 2$ & 4 & $U_{d c}$ & 0 & 0 \\
\hline$t_{3} \sim t_{4}$ & $-U_{d c} / 4$ & 3 & $3 U_{d c} / 4$ & 1 & $U_{d c} / 4$ \\
\hline$t_{4} \sim t_{5}$ & 0 & 2 & $U_{d c} / 2$ & 2 & $U_{d c} / 2$ \\
\hline$t_{5} \sim t_{6}$ & $U_{d c} / 4$ & 1 & $U_{d c} / 4$ & 3 & $3 U_{d c} / 4$ \\
\hline$t_{6} \sim t_{7}$ & $U_{d c} / 2$ & 0 & 0 & 4 & $U_{d c}$ \\
\hline$t_{7} \sim t_{8}$ & $U_{d c} / 4$ & 1 & $U_{d c} / 4$ & 3 & $3 U_{d c} / 4$ \\
\hline$t_{8} \sim t_{9}$ & 0 & 2 & $U_{d c} / 2$ & 2 & $U_{d c} / 2$ \\
\hline
\end{tabular}

The specific working process is shown in the analysis in Table 1. The number of sub-modules put into the upper and lower bridge arms are expressed as $N \_$upper and $N$ lower, and the voltages of the upper and lower bridge arms are expressed as $U_{-}$upper and $U_{-}$lower.

In this paper, the carrier phase shift pulse width modulation method is selected for control. The carrier phase shift modulation method is mainly used to select different phase carriers for each sub-module of the MMC bridge arm, spaced $1 / \mathrm{N}$ carrier periods in turn. The threephase modulating wave is a sine wave with $2 \pi / 3$ phase angle difference in sequence, and the modulating waves of the upper and lower bridge arms are inverted in phase.

\subsection{Switching function model}

The MMC switching function model directly reflects the MMC system sub-module inputs and is directly relevant to the analysis of several electrical quantities such as bridge arm voltage, bridge arm current, and sub-module switch transistor current.

Based on the operating status of the MMC submodule, it can be concluded that:

$$
S_{l j_{-} n}= \begin{cases}0, & \left(T_{1} \text { is off }, T_{2} \text { is triggered }\right) \\ 1, & \left(T_{1} \text { is triggered }, T_{2} \text { is off }\right)\end{cases}
$$

Where: $l$ denotes the A, B, C phase unit, $j$ denotes the upper or lower bridge arm, and $n$ denotes the sub-module of $1 \sim \mathrm{N}$.

Assuming that the voltage of each submodule is equal to $u_{S M}$, the switching function of all input submodules of the bridge arm is summed to obtain the bridge arm voltage, which can be expressed as:

$$
u_{l j}=\left(\sum_{n=1}^{N} S_{l j_{-} n}\right) u_{S M}
$$

Where: $u_{l j}$ is the voltage of the $j$ bridge arm of the $l$ phase. The capacitor current for the nth submodule of the $j$ bridge arm of the $l$ phase can be expressed as:

$$
i_{c, l j_{-} n}=S_{l j_{-} n} \times i_{l j}
$$

Where $i_{l j}$ is the current in the $j$ bridge arm of the $l$ phase. The bridge arm current can be expressed as:

$$
i_{l j}=\frac{\sum_{n=1}^{N} i_{c, j_{\_} n}}{\sum_{n=1}^{N} S_{l j_{-} n}}
$$

\section{MMC dynamic mathematical model and decoupling controller}

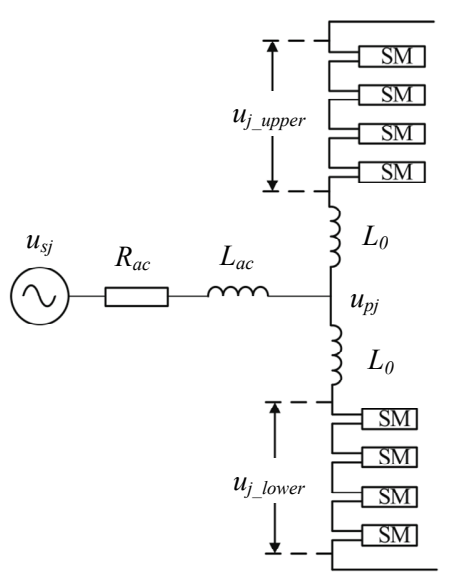

Fig. 2. MMC dynamic mathematical model equivalent circuit diagram

The MMC dynamic mathematical model equivalent circuit diagram (shown in Figure 2) is represented by a three-phase system equivalent to a one-phase system. $u_{s j}$ is the AC power supply, $R_{a c}$ is the connection resistance, $L_{a c}$ is the connection inductor, $u_{p j}$ is the MMC port voltage, $L_{0}$ is the bridge arm inductor, $u_{j_{\_} \text {upper }}$ is the upper bridge arm voltage, $u_{j}$ lower is the lower bridge arm voltage, and $j$ denotes phases $\mathrm{a}, \mathrm{b}$, and $\mathrm{c}$.

The $\mathrm{AC}$ side differential equation for the MMC system in the abc coordinate system can be obtained from Figure 3:

$$
\left[\begin{array}{l}
u_{s a} \\
u_{s b} \\
u_{s c}
\end{array}\right]-\left[\begin{array}{l}
u_{p a} \\
u_{p b} \\
u_{p c}
\end{array}\right]=L \frac{d}{d t}\left[\begin{array}{l}
i_{a} \\
i_{b} \\
i_{c}
\end{array}\right]+R\left[\begin{array}{c}
i_{a} \\
i_{b} \\
i_{c}
\end{array}\right]
$$




$$
\begin{gathered}
L=L_{a c}+L_{0} \\
u_{p j}=\frac{1}{2}\left(u_{j_{-} \text {lower }}-u_{j_{-} \text {upper }}\right)
\end{gathered}
$$

The Pike transformation of equation (5) transforms the MMC AC side differential equation in the threephase stationary coordinate system to the dq axis. we get:

$$
\begin{aligned}
& u_{s d}-u_{p d}=-L \frac{d i_{d}}{d t}-R i_{d}-\omega L i_{q} \\
& u_{s q}-u_{p q}=-L \frac{d i_{q}}{d t}-R i_{q}+\omega L i_{d}
\end{aligned}
$$

In equation (11) and (12), $\omega L i_{d}, \omega L i_{q}$ represent the decoupling terms of the dq axis.

Through the Laplace transform of equations (8) and (9), we can get:

$$
\begin{aligned}
& u_{s d}(s)-u_{p d}(s)=-(R+L s) i_{d}(s)-\omega L i_{q}(s) \\
& u_{s q}(s)-u_{p q}(s)=-(R+L s) i_{q}(s)+\omega L i_{d}(s)
\end{aligned}
$$

If we set :

$$
u_{d}(s)=(R+L s) i_{d}(s), u_{q}(s)=(R+L s) i_{q}(s)
$$

Can get:

$$
\begin{aligned}
& u_{d}(s)=-u_{s d}(s)+u_{p d}(s)+\omega L i_{q}(s) \\
& u_{q}(s)=-u_{s q}(s)+u_{p q}(s)+\omega L i_{d}(s)
\end{aligned}
$$

Thus, the controller schematic for controlling $u_{p d}$ and $u_{p q}$ can be built, as shown in Figure 3. The inner and outer loop controller of the three-phase MMC system consists of two parts, the inner loop controller and the outer loop controller. The inner loop controller refers to the current-loop controller in the text, through which the current-loop controller achieves fast and effective tracking of the current reference value. The outer loop controller is divided into active control quantity control and reactive control quantity control. The active control quantity of the outer loop is selected for constant DC voltage control and the reactive control quantity is selected for constant reactive power control. Combined with the inner loop controller of the MMC system, the double closed-loop control of the three-phase MMC system can be obtained, and the MMC inner and outer loop controller is used to control the MMC system. The modulating wave is generated by the action of the MMC inner and outer loop controller, and then the trigger and shutdown of each sub-module is controlled by the carrier phase shift pulse width modulation. Finally, the start-up and operation requirements of the sub-module of the MMC system are realized.

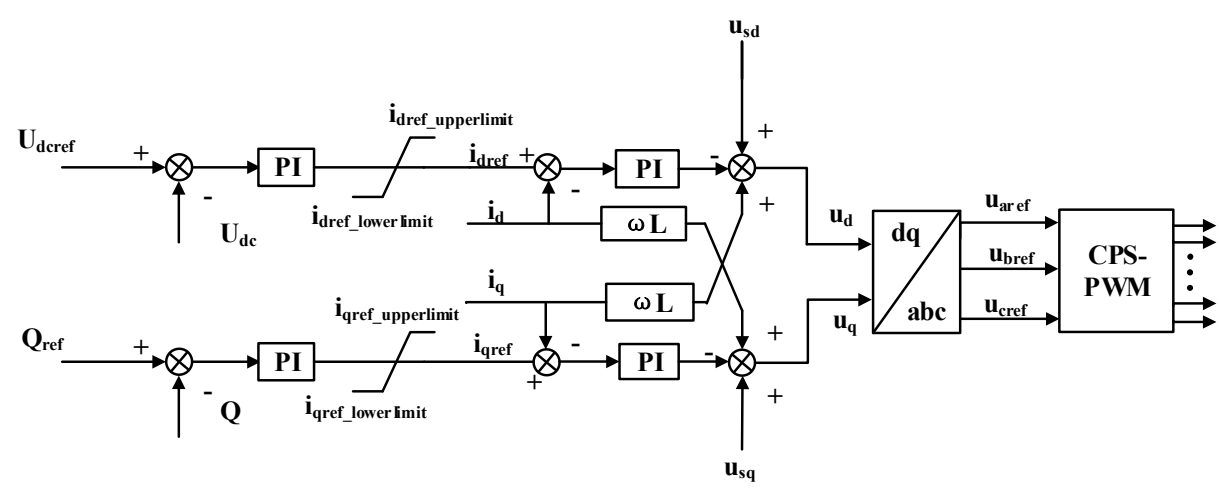

Fig. 3. Schematic diagram of the inner and outer loop controller of the three-phase MMC system

\section{MMC pre-charge}

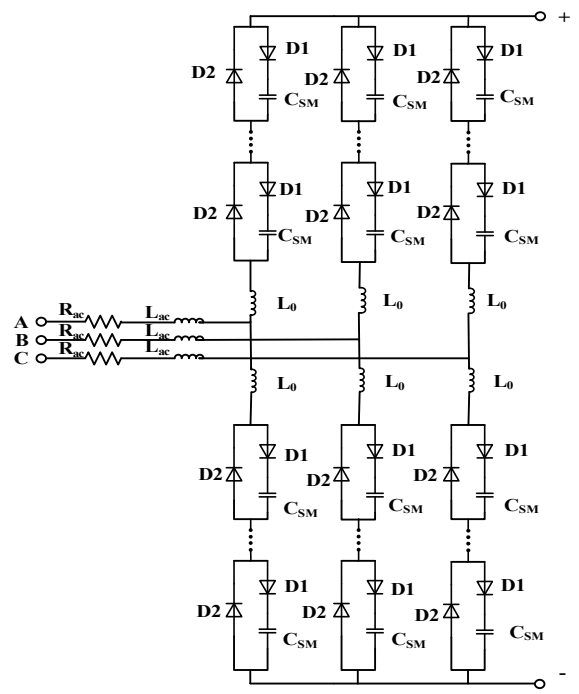

Fig. 4. Uncontrolled charging equivalent circuit diagram
The MMC pre-charging method is chosen as uncontrolled charging, the IGBTs of the sub-module are all in the off state. And the pre-charging of the submodule capacitors is completed by the AC system, the equivalent circuit diagram is shown in Figure 4. Uncontrolled charging means that the AC system line voltage charges the capacitors of the submodule. We can get:

$$
u_{S M}=\frac{\sqrt{3} U_{p h}}{N}
$$

Where: $U_{p h}$ represents the phase voltage of the AC system.

Calculated by the DC voltage when it is stable, the voltage of the sub-module is:

$$
u_{S M}{ }^{\prime}=\frac{U_{d c}}{N}
$$

Where: $U_{d c}$ represents the DC side voltage when it is stable.

From the relationship between the effective value of AC phase voltage and DC side voltage, the following results can be obtained: 


$$
u_{p h}=\frac{m U_{d c}}{2}
$$

Where: $m$ is the modulation ratio. $m$ is generally less than or equal to 1 .

From equation (14), (15) and (16), we can get that $u_{S M}$ is less than $u_{S M}$. That is, the maximum value of uncontrolled charging to sub-module capacitance charging is generally less than the sub-module voltage value required for system operation. And uncontrolled charging cannot charge to the capacitor voltage of the system in stable operation. Uncontrolled pre-charging can only achieve a certain degree of the system precharging, but it avoids the system from entering the startup state when the capacitor voltage is 0 , which greatly reduces the power shock of the system start-up process. After the uncontrolled charging is completed, the MMC can be controlled by the inner and outer loop controller of the three-phase MMC system to complete the start-up.

\section{Simulation experiment verification}

The experimental parameters are set as follows: $\mathrm{AC}$ system voltage $U_{a c}=380 \mathrm{~V}, \mathrm{AC}$ system frequency $f_{s}=50 \mathrm{HZ}$, connected inductor $L_{a c}=1 \mathrm{mH}$, connected resistance $R_{a c}=1 \Omega$, number of sub-modules $\mathrm{N}=4$, submodule capacitor value $C_{S M}=5000 \mu \mathrm{F}$, bridge arm inductor $L_{0}=1 \mu \mathrm{H}$, carrier frequency $f_{k}=1 \mathrm{kHZ}$. The simulation results are shown in Figure 5 Figure 9.

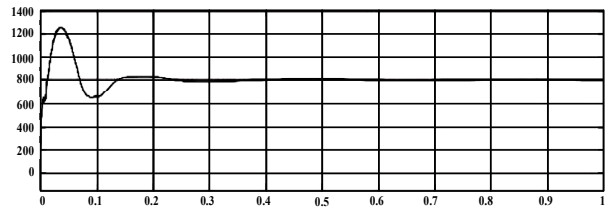

Figure 5 MMC DC-side voltage

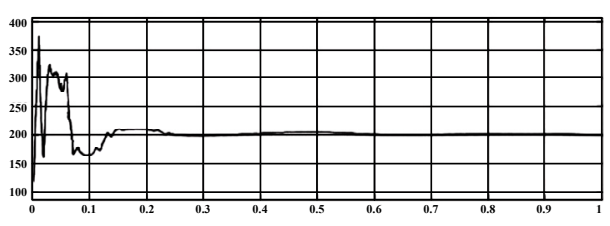

Figure 6 MMC sub-module voltage

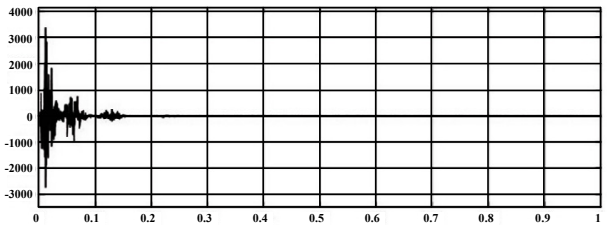

Figure 7 A phase bridge arm current

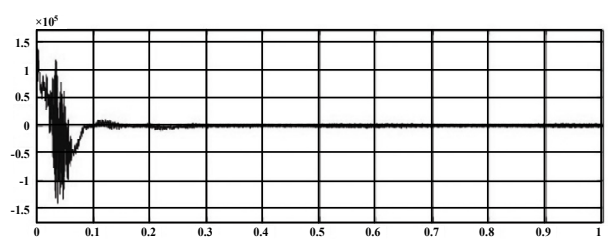

Figure 8 MMC AC side reactive power

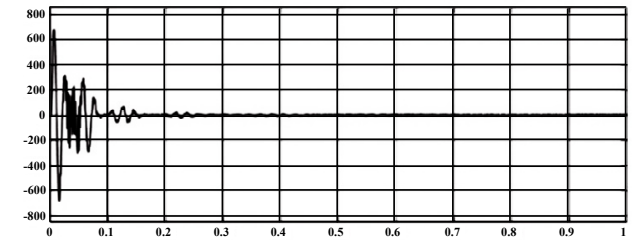

Figure $9 \mathrm{MMC}$ AC side phase A current

The DC-side voltage starts to rise from the initial uncontrolled charging voltage of about $540 \mathrm{~V}$, and the controller finally controls the DC-side voltage to stabilize at $800 \mathrm{~V}$, as shown in Figure 5. The sub-module voltage is also stabilized at $200 \mathrm{~V}$ by the controller action, as shown in Figure 6. The bridge arm current is regulated from the beginning of a certain current shock regulation to 0, as shown in Figure 7. After controlling the controller regulation action, the reactive power is finally regulated to near 0 , as shown in Figure 8 . The A phase currents of the $\mathrm{MMC}$ are also regulated to 0 from the beginning of the fluctuation state, as shown in Figures 9.

\section{Conclusion}

Based on the topology, working principle and switching function of the modular multilevel converter, the mathematical model of the dynamic characteristics of the modular multilevel converter is derived. After introducing the control quantities, the controller based on the modular multi-level converter is established. Simulation experiments are performed to first complete the target of pre-charging by uncontrolled pre-charging, and then controls the start-up of the modular multilevel converter by the established controller. The simulation results show that the control method in this paper satisfies the start-up control requirements of DC side voltage, sub-module voltage, bridge arm current, reactive power and $\mathrm{AC}$ system current. At the same time, the energy impact during the start-up process is reduced, and the smooth start-up of the modular multilevel converter is realized.

\section{Acknowledgments}

The research in this paper was supported by Research on Energy Systems of Smart Park. Research and Demonstration on Key Technologies of Multi-functional Energy Router of Shanghai Science and Technology Commission (18DZ1203700).

\section{References}

1. Kurtoglu, M., Eroglu, F., Arslan, A. O., \& Vural, A. M.. Recent contributions and future prospects of the modular multilevel converters: a comprehensive review. International Transactions on Electrical Energy Systems, 29(3), e2763.1-e2763.36. (2019)

2. Shuai, S., Jiang, M., Zhang, J., \& Wu, X.. A capacitor voltage balancing method for a modular multilevel dc transformer for de distribution system. 
IEEE Transactions on Power Electronics, PP(99), 11. (2017)

3. Yang, S., Yi, T., \& Peng, W.. Distributed control for a modular multilevel converter. IEEE Transactions on Power Electronics, 33(7), 5578-5591. (2017)

4. Shi, X., Liu, B., Wang, Z., Li, Y., Tolbert, L. M., \& F Wang. Modelling, control design and analysis of a startup scheme for modular multilevel converters. IEEE Transactions on Industrial Electronics, 62(11), 7009-7024. (2015) 